\title{
On mooring line tension and fatigue prediction for offshore vertical axis wind turbines: a comparison of lumped-mass and quasi-static approaches
}

D. Cevasco ${ }^{1}$, M. Collu ${ }^{2}$, C.M. Rizzo ${ }^{3}$ and M. Hall ${ }^{4}$

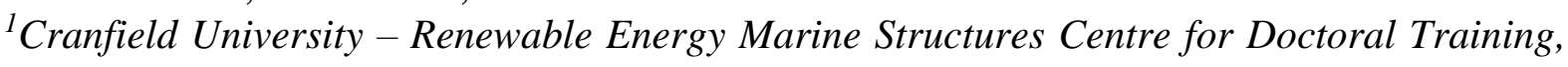
United Kingdom,d.cevasco@cranfield.ac.uk

${ }^{2}$ Cranfield University - Offshore Renewable Energy Engineering Centre, United Kingdom, maurizio.collu@cranfield.ac.uk

${ }^{3}$ DITEN - Marine Structures Testing Lab, Università degli studi di Genova, Italy, cesare.rizzo@unige.it

${ }^{4}$ University of Prince Edward Island - School of Sustainable Design Engineering, Canada, mthall@upei.ca

\begin{abstract}
Despite several potential advantages, relatively few studies and design support tools have been developed for floating VAWTs. Due to the substantial aerodynamics differences, the analyses of VAWT on floating structures cannot be easily extended from what have been already done for HAWTs. Therefore, the main aim of the present work is to compare the dynamic response of the FOWT system adopting two different mooring dynamics approaches. Two version of the in-house aero-hydro-mooring coupled model of dynamics for VAWT (FloVAWT) have been used, using: a mooring quasi-static model, which solves the equations using an energetic approach, and a modified version of FloVAWT, which instead couples with the lumped mass mooring line model MoorDyn. The results, in terms of mooring line tension, fatigue and response in frequency have been obtained and analysed, based on a 5MW Darrieus type rotor supported by the OC4-DeepCwind semisubmersible.
\end{abstract}

\section{Introduction}

Since the world's first installation of a floating large-capacity wind turbine in deep waters (Hywind project, 2009), the interest in offshore floating wind has been substantially growing. Various concepts and prototypes have since been deployed [1], and now the industry has progressed to the approval and development of offshore floating wind farms. Representing a new energy opportunity also for countries lacking shallow coastal waters and rich in wind resources, a number of studies and internationally coordinated research activities have been conducted on the modelling and analysis of the dynamics of offshore floating Horizontal Axis Wind Turbine (HAWT) systems [2,3].

However, the growing desire for more cost-effective wind farms is pushing this investigation further, reconsidering Vertical Axis Wind Turbines (VAWT) [4]. Thanks to their effective advantages when employed for floating platforms [4,5], various floating VAWTs concepts have been proposed (such as DeepWind, NOVA and inFLOW projects [6-8]). Therefore, fully coupled aero-hydro-servoelastic dynamic models were developed to assess their feasibility and better quantify and identify their pros and cons compared to the already employed HAWTs concepts [9-11].

\subsection{Problem statement}

Due to the substantial differences between HAWT and VAWT aerodynamics, the analyses on floating HAWT cannot be easily extended to floating VAWT systems, and new codes have to be developed or adapted ad-hoc for these new kind of structures [12]. The fundamental challenge of 
these design tools is to be quick and flexible, to be used for analysing the large number of load and configuration cases, but being able to capture all the main important dynamics.

In particular, the dynamic response and station keeping modelling of VAWT systems, subject to a larger yaw moment, is one of the novel aspects to be represented. Several studies and code-tocode comparisons (Borg [13], Cheng [14]) have been conducted for what concerns the aero-hydroelastic models of dynamics, to understand the most suitable models and effects necessary to account for when modelling these structures, depending on their specific designs (turbine and platform) and operational conditions. However, no extensive analysis of mooring systems models for floating VAWT configurations has been conducted, in order to assess when simplified static approaches can lead to satisfactory results, and when it is necessary instead to adopt higher fidelity codes.

\subsection{Aim and Methodology}

Starting from a systematic comparative analysis of platform motions and mooring loading conditions for worsening metocean conditions [15], a comprehensive study on the fatigue assessment and phenomena playing an important role in the tension of the mooring chain is carried out. Therefore, the purpose of this paper is to support and complete the recommendations on when it is necessary to adopt a more accurate dynamic modelling approach than a static one for catenary mooring lines of floating VAWTs, aiming to reach a good compromise between computational time and accuracy.

First, a brief recap of the coupled model of dynamics code (FloVAWT) is presented, summarizing the program capabilities and describing the mooring model of dynamics adopted. Then, a comparison of the results obtained adopting the two mooring dynamics models are reported in terms of realisations-averaged tensions in, and cumulative damages of, the mooring lines. Finally, a more in-depth view of the system response (motion response and mooring line fatigue) and the energy distribution in frequency is shown through a spectral analysis.

\section{Coupled model of dynamics: FloVAWT}

\subsection{FloVAWT recap}

FloVAWT (Floating Vertical Axis Wind Turbines) is a coupled dynamics design tool implemented in MATLAB/Simulink, currently being developed (at Cranfield University) to support the development of the preliminary design stages of offshore floating VAWTs. More detailed information about development and verification of FloVAWT has been reported by Collu et al. [1519], however a summary of its capabilities are here given.

Starting from the aerodynamics, the Double Multiple Streamtube (DMS) momentum model implemented has been validated in semi-coupled simulations showing, despite its limitations, a good agreement for predicted power against experimental data for a number of VAWT systems, over a wide range of operating conditions [20]. Additionally, accounting for corrections for dynamic stall (Gormont-Berg model), tower shadow and tip losses, and enabling to simulate turbulent incident wind (generated by TurbSim [21]), the aerodynamic module is able to catch variation in the Reynolds number and considers the modification for the induction factor due to the platform motions (validated by Collu et al. [22], against experimental data obtained for an inclined VAWT by Mertens [23]).

For the hydrodynamics, the Cummins' equation is used, and SESAM/Wadam is run to derive the frequency-dependent first-order wave excitation and mean drift forces, as well as the hydrodynamic coefficients, which are then imported into the MATLAB environment. In particular, a radiation-force state-space approximation, based on the Marine Systems Simulator toolbox [24], is implemented to enhance computational efficiency of the Simulink model. The simulated sea surface elevation time series for each load case and related wave loads on the structure are generated using 
an IFFT approach as presented by Jonkman [25] (Collu et al. [18]). Additionally a linear viscous damping model, applying a fraction of critical damping in each DOF is used. Firstly validated against an offshore oil and gas semisubmersible platform [20], the hydrodynamic model was further validated against the OC4-DeepCwind employed in the current work [22].

Regarding the structure dynamics, FloVAWT makes a rigid body assumptions; nonetheless, it applies analytical formulations to represent the fundamental gyroscopic forces and system moments of inertia $\left(\mathrm{I}_{\mathrm{xx}}\right.$ and $\mathrm{I}_{\mathrm{yy}}$ ) variations due to the rotation of the rotor. Supporting this approach, Wang et al. [26] showed that the structural stiffness of the wind turbine itself does not have a substantial impact on the global motion response.

On the other hand, as outlined by Borg et al [27], where the in-house-code was compared to SIMO-Riflex-DMS simulation tool for floating VAWTs (developed at NTNU), the major discrepancies in the results lay in the lower order damping approach and mooring line model implemented in FloVAWT, over than its lack of a control system for the maximization of the power capture through variable speed rotor operation. While no generator, transmission and control system have been considered yet in this work, and a constant rotor rotational speed $(5.26 \mathrm{rpm})$ is considered, a higher-fidelity mooring system has been implemented to analyse its impact on the global response.

\subsection{Mooring dynamics models}

As observed by Borg in [28], when using quasi-static (QS) models to analyse mooring configurations with discontinuities (e.g. buoyancy and clump weight aids), the numerical stability of the computational procedure becomes an issue. For this reason, to be able to cope with novel FOWT mooring layouts, FloVAWT was integrated with an energy variation principle model, by deriving the mooring line system equations based on the energy transfer within the system. This more robust quasi-static energetic (QSE) computational scheme, which firstly was introduced by Ma et al. [29], have been implemented in Collu al. [17], with the limitation of neglecting the line elasticity.

However, linear and non-linear QS models, although their ease of implementation for both turbine or wind farm level modelling (see e.g.[30]), can lead to inaccurate results in motion and forces along the lines. For severe operating condition, Borg et al. [31] reported high discrepancies in the motions for the linearized force-based catenary model in FloVAWT compared to the FEM approach in Riflex. This observation agrees with what found by Hall et al. [32], who observed that, for different floating HAWTs designs and in specific cases, dynamic FEM modelled mooring system accounted for a significant portion of the total platform damping, leading to alterations in the platform motions. On the other hand, Karimirad [33] observed that the use of QS model, rather than a multi body approach, affects significantly the tension predicted (mainly due to the hydrodynamic damping and inertial forces consideration), not modifying significantly the global displacement.

This premise motivates the study conducted in this work, where the low order lumped mass model of MoorDyn (MD) [34], integrated into FloVAWT [15], is used to capture more accurately the system dynamics of a VAWT system. Although it does not account for wave kinematics and seabed friction interaction, this model has been validated in the FAST fully-coupled simulations, showing a good agreement with experimental data for the 5MW-DeepCwind reference model (Hall et al. [35]).

In particular, the results from its coupling have been compared to the one obtained by the QSE method implementation. The MD model, indeed, shares with it the approximation on the estimation of the static tension, due discretization inaccuracy in evaluating the length of the line portion on the seabed. Following the comparison of these two approaches is carried out, outlining the differences in the system dynamic response but focusing on the discrepancies of the predicted tension. 


\section{FOWT system and load cases definition}

\subsection{Turbine and platform characteristics}

This work presents the response of a 5MW Darrieus type rotor [36], supported by the OC4DeepCwind semisubmersible, rearranging the ballast to achieve the same draft of the floating HAWT configuration (as described by Wang et al. [37]). The mooring line layout and characteristics are the one suggested by the OC4 phase II and already implemented in [15]: three catenary lines spread symmetrically about the vertical axis, each connected to one column of the support structure.

The parameters were not tuned for this particular work, since this kind of wind turbine was used before by the authors $[15,22,27]$. The main characteristics of both the rotor and the support structure (including wind turbine inertia) are given in Figure 1, together with a conceptual visualisation of the FOWT layout.

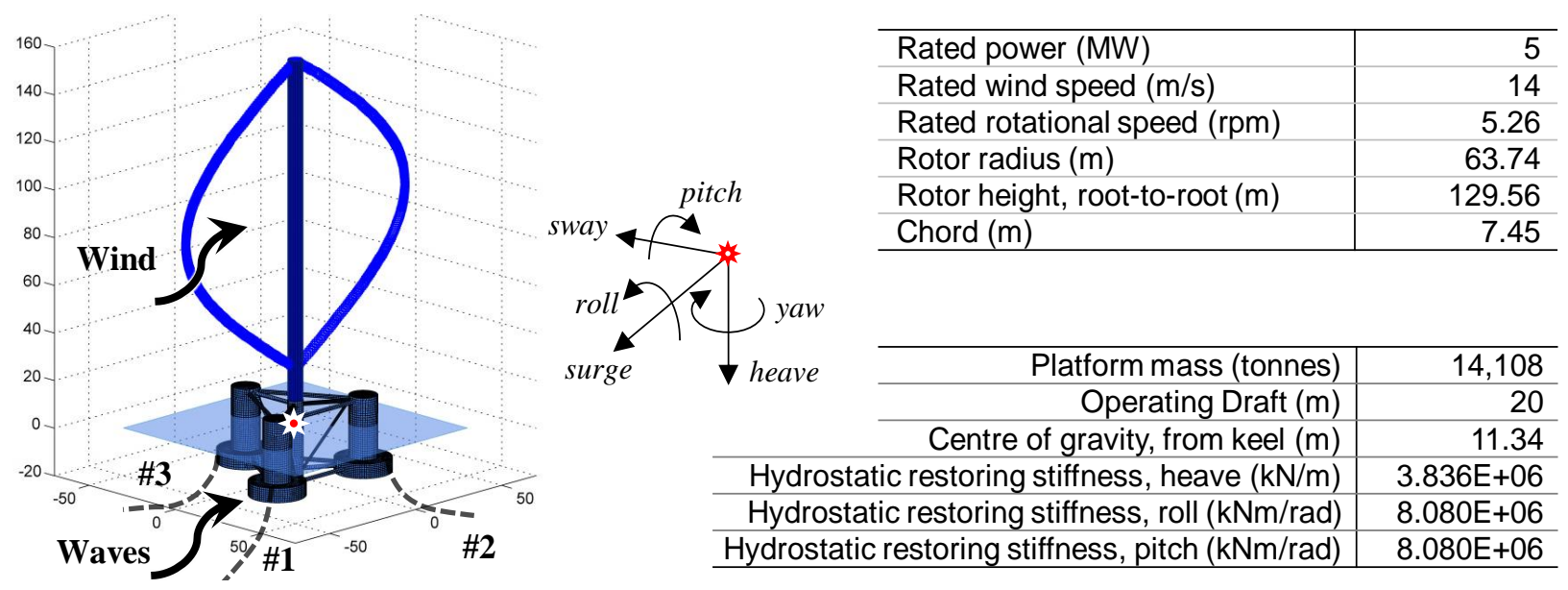

Figure 1: Conceptual visualization of the FOWT investigated and main characteristics

\subsection{Load cases and environmental conditions}

A series of load cases have been considered (Table 1), with stochastically generated turbulent wind speeds (named either LC or LC\#T) and correlated irregular waves time histories (JONSWAP wave spectrum model), both aligned along the x-direction (Figure 1). For each load case, five realisations of one hour (changing wind and wave random seeds) have been performed in the time domain, with $0.1 \mathrm{~s}$ time step. For this reason, the results are presented as average among the realisations, characterised by mean value and standard deviation (std). In particular, the time signals of the mooring tension, at the fairlead, have been then analysed to highlight the differences between the two models. Finally, a prediction of the cumulative damage on element of the mooring chain at the fairlead is presented, highlighting the impact of the adopted modelling approach on the fatigue life estimation.

Table 1: LCs from below rated (left), up to the cut-out wind speed (right)

\begin{tabular}{|c|c|c|c|c|c|}
\hline LC\#T & $\begin{array}{c}\mathrm{U}_{\text {hub }} \\
(\mathrm{m} / \mathrm{s})\end{array}$ & $\begin{array}{c}\mathrm{H}_{\mathrm{S}} \\
(\mathrm{m} / \mathrm{s})\end{array}$ & $\begin{array}{c}\mathrm{T}_{\mathrm{p}} \\
(\mathrm{s})\end{array}$ & $\begin{array}{c}\text { Turbine } \\
\text { status }\end{array}$ & $\begin{array}{c}\mathrm{TI} \\
(\%)\end{array}$ \\
\hline 4.2 & 8 & 2.55 & 9.86 & Operating & 17 \\
4.3 & 10 & 2.88 & 9.98 & Operating & 16 \\
4.4 & 12 & 3.24 & 10.12 & Operating & 15 \\
4.5 & 14 & 3.62 & 10.29 & Operating & 14 \\
\hline
\end{tabular}

\begin{tabular}{|c|c|c|c|c|c|}
\hline LC\#T & $\begin{array}{c}\mathrm{U}_{\text {hub }} \\
(\mathrm{m} / \mathrm{s})\end{array}$ & $\begin{array}{c}\mathrm{H}_{\mathrm{S}} \\
(\mathrm{m} / \mathrm{s})\end{array}$ & $\begin{array}{c}\mathrm{T}_{\mathrm{p}} \\
(\mathrm{s})\end{array}$ & $\begin{array}{c}\text { Turbine } \\
\text { status }\end{array}$ & $\begin{array}{c}\mathrm{TI} \\
(\%)\end{array}$ \\
\hdashline 4.6 & 18 & 4.44 & 10.66 & Operating & 13 \\
4.7 & 22 & 5.32 & 11.06 & Operating & 12 \\
4.8 & 25 & 6.02 & 11.38 & Operating & 12 \\
4.9 & 25.1 & 6.02 & 11.38 & Shutdown & 12 \\
\hline
\end{tabular}




\section{Preliminary analysis: tension and fatigue of the mooring lines}

\subsection{Tension results and analysis}

After having shown that, for worsening metocean conditions, MD dynamic approach is recommended if the interest is in modelling the translational displacements of the platform [15], a further analysis is here developed to analyse this behaviour, understanding its physical causes and to better analyse which phenomena play an important role in the estimation of the mooring line tension.

Besides the dependency on the wave excitation, it is noticeable (Figure 2) that the tension along the mooring lines is strongly related to the combined effect of aerodynamic trust force and rotation of the rotor. These wind forces affect the line tension increasing the average value of the front chain whilst reducing the one of the back and symmetrically placed lines. In support to this observation, the tension in all the three lines is shown to get values close to the starting pre-tension condition at the shutdown of the turbine (around $1200 \mathrm{kN}$ and $1010 \mathrm{kN}$ for QSE and MD, respectively).

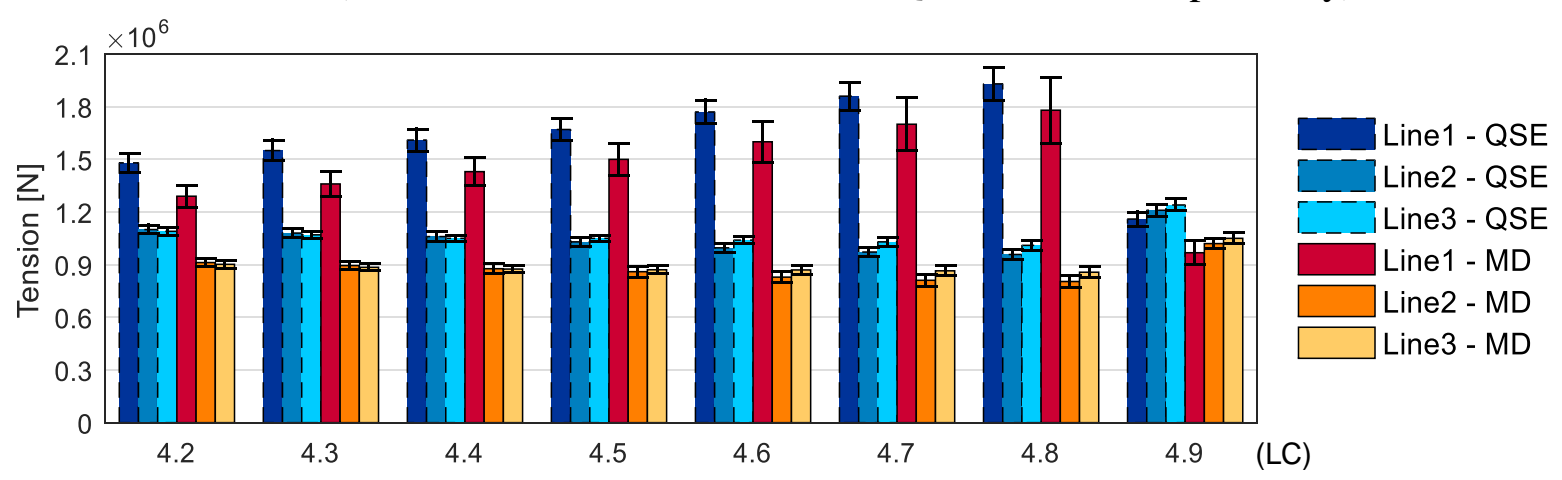

Figure 2: Mooring lines tension among LCs

By comparing the two modelling approaches, it is observable that the tensions estimated by the MD model have lower magnitudes and, generally, higher standard deviation across the LCs, compared to the QSE model. The fact that generally the length of the line suspended in the MD approach is lower than in the QSE approach (Figure 3-right), because part of the energy is dissipated in the elastic deformation of the lines in the MD model, leads, indeed, to lower tensions. On the other hand, the inclusion of hydrodynamic damping and inertial forces in the MD adds new dynamic loads, which cause higher tensions fluctuation: this effect is observable in the higher std for the overtensioned front line (Figure 2), as well as in the global restoring response of the mooring system on the platform in the surge motion, where a strong dependence on local acceleration and velocities is noticeable (Figure 3-left).

Henceforth, the focus will be on the analysis of the mooring front line (line 1), as the wind and wave are aligned longitudinally.
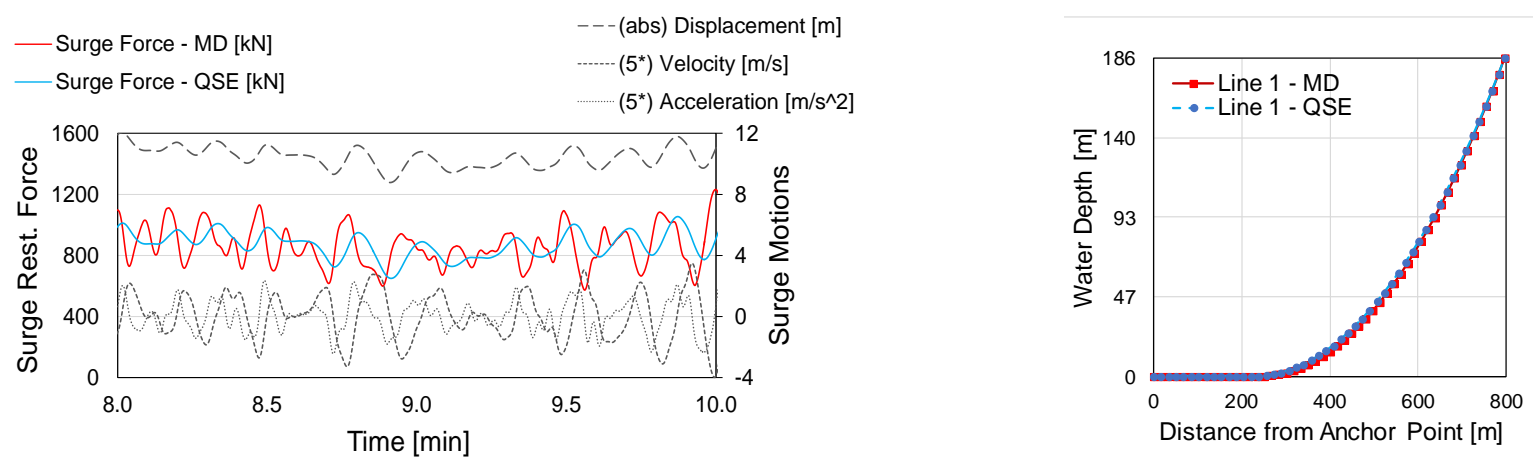

Figure 3: QSE vs MD secondary comparisons: time histories (LC4.6T) of the surge restoring force and surge motion (left) and initial nondisplaced position layout of the front line (right) 


\subsection{Cycle counting methods and fatigue estimation}

The hour-damage of the first studless chain link from the fairlead has been calculated for each realization, applying Miner's cumulative damage rule. By converting the stress time histories into cycles at different stress ranges, the maximum number of cycle, per stress range, tolerated by the material and geometry have been estimated through S-N curve for open links suggested by DNVGLOS-E301 [38]. Different cycle counting methods are possible, and some of most used peak counting and range counting methods have been employed, due their ease of implementation and fidelity in the representation of simple sinusoidal signals, respectively [39].

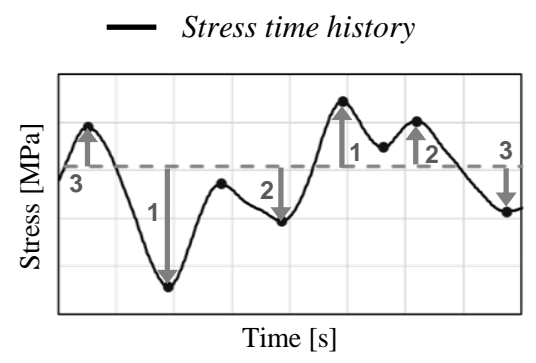

(a)

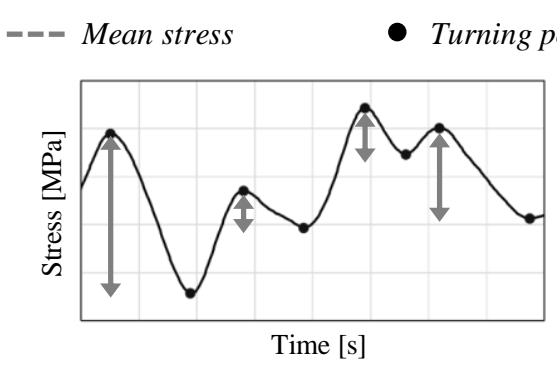

(b)

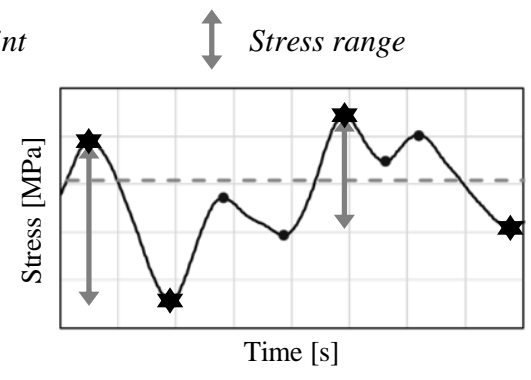

(c)

Figure 4: Cycle counting methods' schematics: a) PC employed, matching the local maxima/minima for the stress range determination; b) PVC; c) MCPC

After reducing the signal into turning point, one of the simplest method suggests to identify one-cycle stress ranges considering all local maxima and pair them with fictitious same-amplitude local minima $[39,40]$. Not taking into account the real draughts of the signal, and thus effective stress ranges, this method has been applied in its modified version, according to what suggested by the ASTM [41]. Aiming to the most conservative estimation, the maximum local maxima above the mean have been allocated to the minimum local minima, continuing then to pair all the other peaks and valleys accordingly, in descendent order. The second selected technique, still within the peak counting category, was the well-known Peak-Valley Counting method (PVC) [40]. Furthermore, to better understand the contribution of small stress ranges in the highly oscillatory tension signals, a variation of the first method has been considered [41]: only the maximum (minimum) of the local peaks (valleys) for each mean crossing (MC Peak Counting) band were selected for the identification of the local range, cutting out the majority of the small ranges and theoretically setting the results between the two previously described methods (Figure 4). Finally, the widely applied Rainflow Counting (RFC) method, in the codified version suggested by the ASTM [41], have been applied to complete the study with a range counting method.

Despite the lower averaged tension in the mooring lines, the additional effects evaluated by the MD approach lead to an order of magnitude higher fatigue damage (Figure 5-right), compared to the QSE model. By comparing the results obtained with the various counting methods (Figure 5-left), it is noticeable that the over-conservative PC method could be accepted for MD, but cannot be accepted when using a QSE approach (Figure 5-top left). While for the former (MD) it forecasts an additional estimated damage from $70 \%$ to $20 \%$ higher than the other methods, it is characterised by an unpredicted high variance among the LCs realisations for the latter (QSE). On the other hand, MCPC, PVC and RFC methods show a good agreement, incrementing both damage mean value and std for more severe conditions, with relative percentage difference of about $30 \%$ for rated and above rated operational conditions. Therefore an easier peak counting method (either MCPC or PVC) could be applied for the estimation of the damage. However, the use of RCF approach is suggested, leading to more conservative results. Furthermore, the negligible disagreement between MCPC and PVC 
methods demonstrate that the small ranges cycles are not the cause of the discrepancy of the two mooring modelling approaches.

Finally, as observed for the tension, the aerodynamics loads' oscillations have a strong effect on the mooring lines damage. In particular, the dynamic model is more sensitive to the wind loads, since the damage is about an order of magnitude lower when the wind turbine is not operating (cutout case).
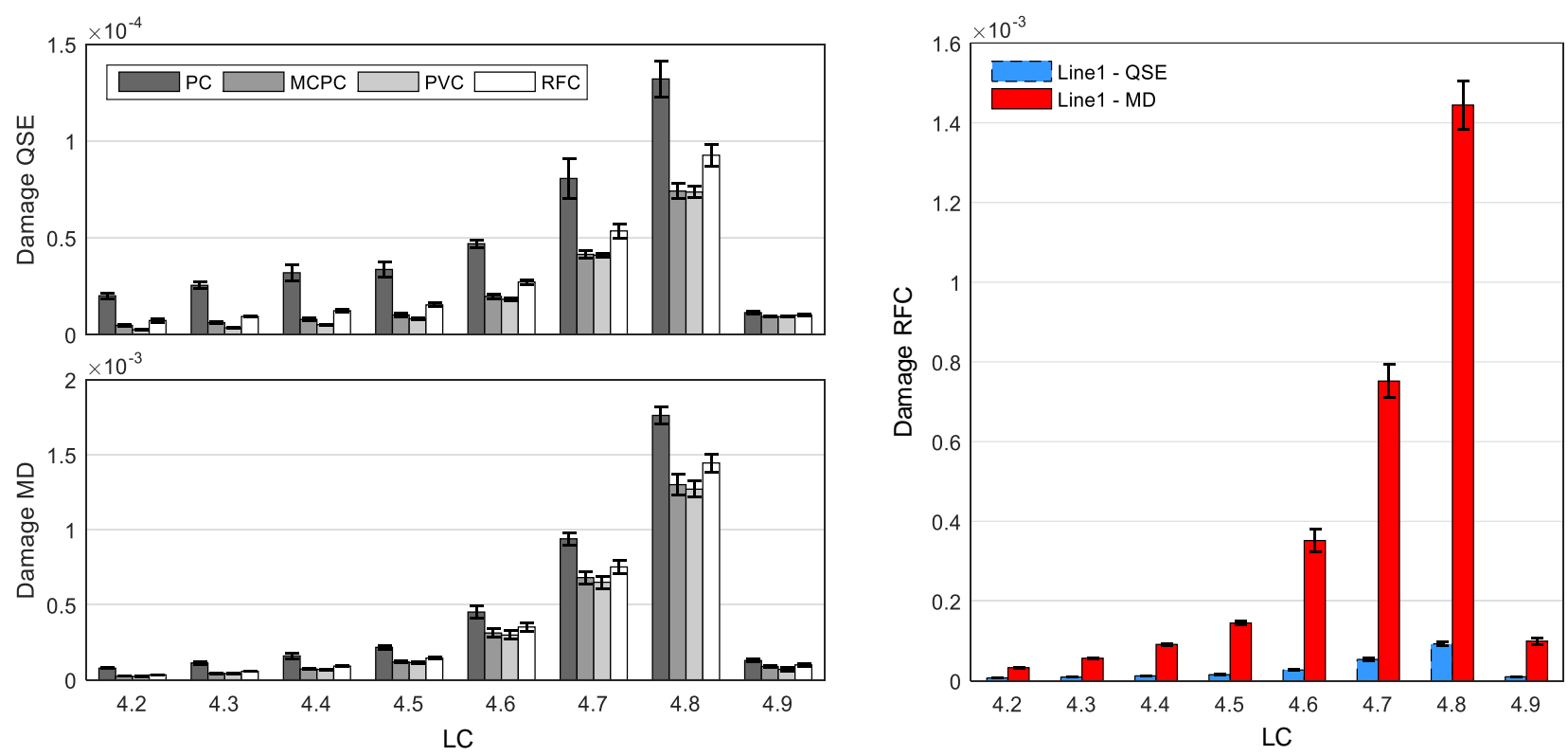

Figure 5: Hour-damage estimation in line 1, by comparing: the results from the different counting methods (left) and the ones obtained from QSE and MD by adopting RFC method (right)

\section{Spectral analysis: results and discussion}

A spectral analysis of the tension time signals has been performed to understand the main physical phenomena impacting on these forces (see Sec.4.1). The power spectral density (PSD) plots of the tension at the fairlead (of the upwind line) were generated using the Fast Fourier Transform technique, after de-trending the time signals, to be then averaged among the realisation for each LC. Similarly, the PSD plots of the main body motions and loads have been derived. However, for brevity, only the results for LCs 4.2 and 4.8 and of the along-pitch aerodynamic load component and platform displacement are reported. Furthermore, the pseudo-RAOs of the line tension have been derived as the square root of the ratio of the tension and the wave spectra response amplitude. The concept of "pseudo-RAO", firstly introduced by Jonkman [42], is here used because the underlying models for the aero- and mooring system dynamics are nonlinear.

Analysing the PSDs of the tension among LCs, it can be noticed that the response increases of an order magnitude passing from the lower sea state case to the most severe one (Figure 6Figure 7Figure 7 centre/right, respectively). By looking at their trend in frequency, instead, the energy peak is shown to move from the wind turbulence spectrum frequencies and platform natural frequency in pitch toward the higher frequencies of wind and wave excitations, for above rated condition (Figure 6Figure 7left). Focusing then on the comparison between the mooring models, a higher energy content in frequency is observable for the MD modelled tension (Figure 6Figure 7, left). This relative difference, already visible in the higher std of the realisation-averaged results (Figure 2), increases for the worsening of the metocean conditions. In particular, it is mainly due to the higher response of the MD to both hydro- and aero-dynamic excitations and loads compared to the quasi-static approach. 
While a good agreement between the MD and QSE can be observed at the very low frequencies (wind turbulence spectrum range) (Figure 6-Figure 7, centre-top), already at below-rated operating condition, the QSE model substantially under-predicts the medium/high frequencies response. On the other hand, at low/medium frequencies (up to $0.05 \mathrm{~Hz}$ ) it over-predicts the excitation peak at the platform pitch resonant response (at $0.034 \mathrm{~Hz}$ ) by about a factor three relative to the dynamic model (Figure 7, centre-top). Additionally, it shows resonant behaviour to the platform surge natural frequency at $0.009 \mathrm{~Hz}$ (Figure 6-Figure 7, centre-top). Oppositely, with the energy moving towards higher frequencies for worsening metocean condition, the MD-modelled line shows a higher response to the first-order wave excitation, in the range of frequencies between the $0.1 \mathrm{~Hz}$ and $0.08 \mathrm{~Hz}$ (Figure 6-Figure 7, top-right). This behaviour can be explained by the lower stiffness of the MD-modelled line, which cause larger motions of the platform [15].

At medium/high frequencies, although the QSE-modelled tension is excited by the operating turbine $2 \mathrm{P}$ and $4 \mathrm{P}$ frequencies, it does not respond to the lower order magnitude aerodynamic loads induced by the platform motions, as already seen by Borg [43], in the range of about $0.05 \mathrm{~Hz}$ around the expected peak responses. This phenomena, which decreases at the turbine shut-down, can be identified as the main cause of the high discrepancy in variance of the tension estimated by the two models (Figure 2), numerically reported in [15]). Furthermore, the plot of the pseudo-RAO of the estimated tensions (Figure 8, left) shows more clearly the strong dependency of MD response from the aerodynamic loads. This behaviour can additionally be tracked in the PSD plot of the pitch displacement, where the high frequency damping of the MD line is observable, mainly at the low wind speed cases (Figure 8, centre-bottom). However, this motion difference seems negligible in the estimation of pitch aerodynamic moment itself (Figure 8, top).
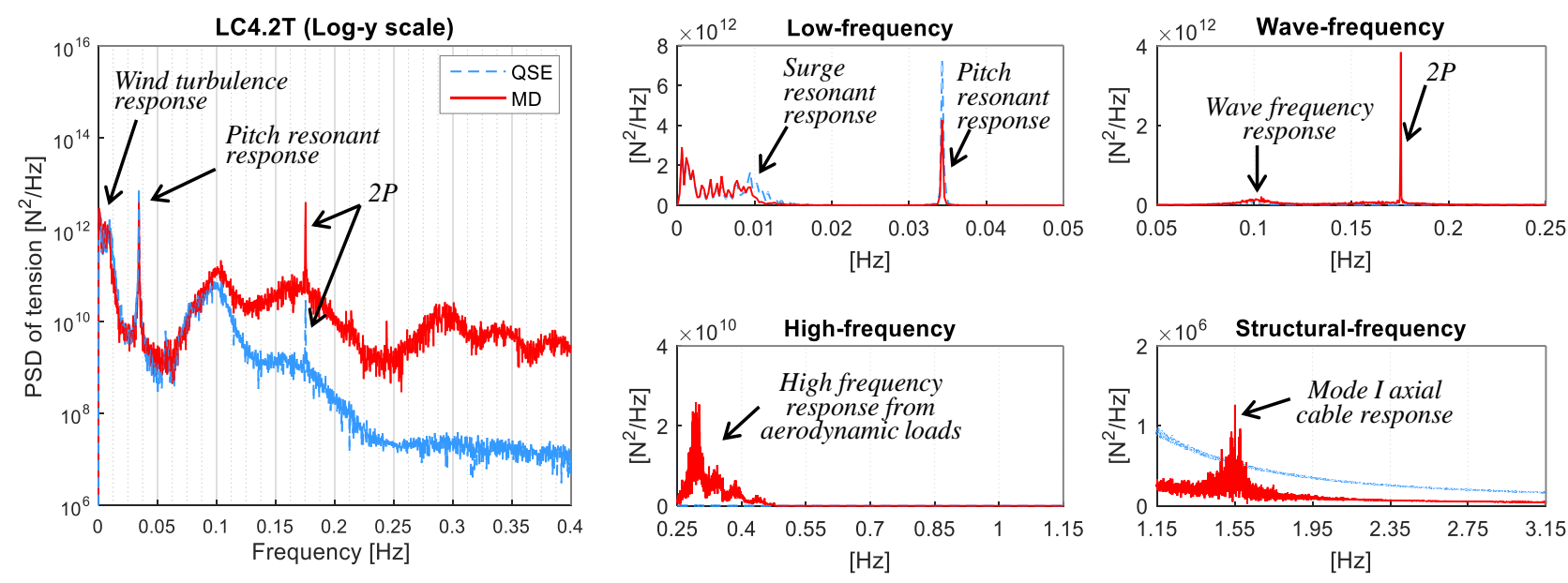

Figure 6: PSD of the line 1 tension for LC4.2T with $U_{w}=8 \mathrm{~m} / \mathrm{s}, \mathrm{H}_{\mathrm{S}}=2.55 \mathrm{~m}$, and $\mathrm{T}_{\mathrm{p}}=9.86 \mathrm{~s}$

At higher frequencies, the dynamic model records the longitudinal cable first vibration mode, observable at $1.54 \mathrm{~Hz}$ and estimated as in [44] (Figure 6Figure 7, top/bottom-left). Although this response increases of an order of magnitude for the most severe operating conditions, it possible to state that the elastic mooring line structural response does not particularly affect the variance of the estimated tension, being the response with smallest order of magnitude. 

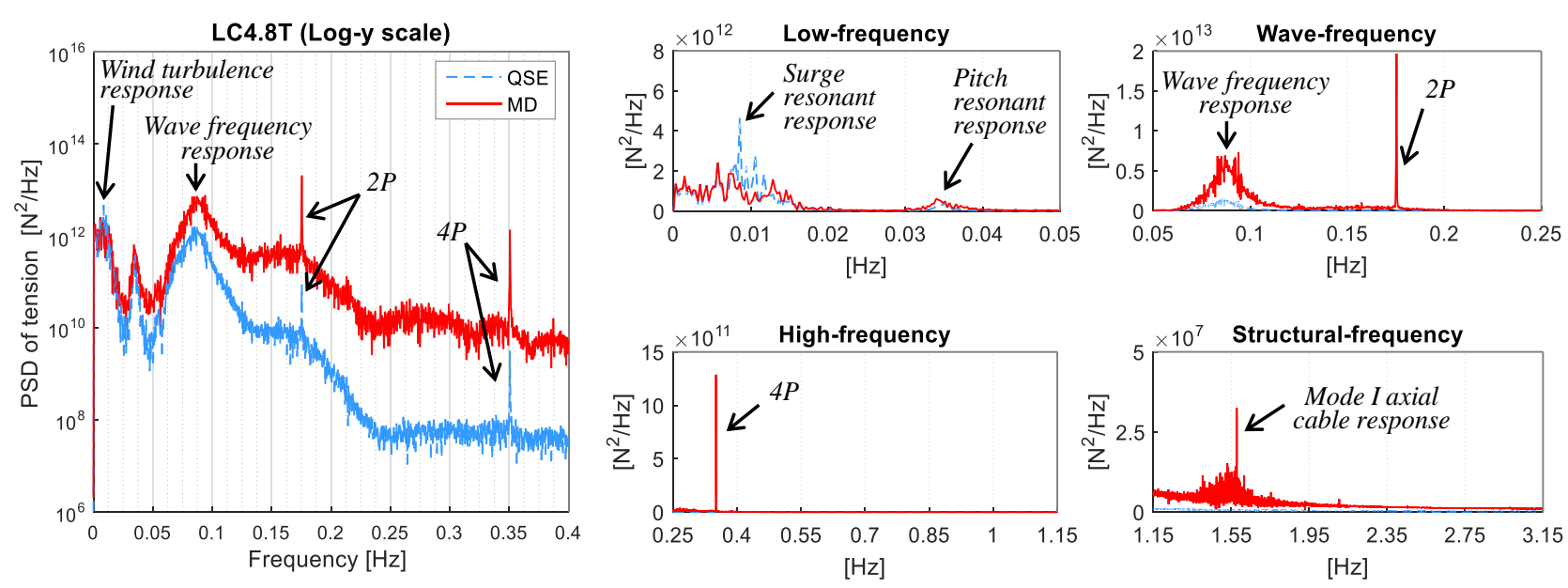

Figure 7: PSD of the line 1 tension for LC4.8T with $\mathrm{U}_{\mathrm{w}}=25 \mathrm{~m} / \mathrm{s}, \mathrm{H}_{\mathrm{S}}=6.02 \mathrm{~m}$, and $\mathrm{T}_{\mathrm{p}}=11.38 \mathrm{~s}$

The MD-FloVAWT simulations here presented show a very good agreement with the results estimated by Chen et al. [45], who modelled and analysed the response of the same floating structure, in Simo-Riflex. Despite Chen's model included structure (blades and tower) elasticity, a constant speed controller for the rated conditions, and a FEM model for the mooring lines, similar energy spectra have been obtained for both body motions and mooring tensions; in particular, the $2 \mathrm{P}$ and $4 \mathrm{P}$ peaks can be observed in the mooring lines response spectra. However, as outlined by Chen [45], this aerodynamics effect on the chains could be relieved by using more advanced control strategies and/or by increasing the number of blades.

On the other hand, differently from what expected and presented by Hall et al. [32] (in the case of a HAWT mounted on the ITI Energy barge support structure), the hydrodynamic drag and added mass forces acting on the mooring lines do not significantly contribute to the platform dynamics. However, this is explained by the fact that, in the present work, the QSE model under-predicts both the tension and the platform translational displacements, since the line axial stiffness is not taken into account, whilst, in the work of [32], the FAST's QS approach modelled the lines elasticity as ProteusDS model, but significantly over-predicted of the platform motions by neglecting the higher order dynamic effect.
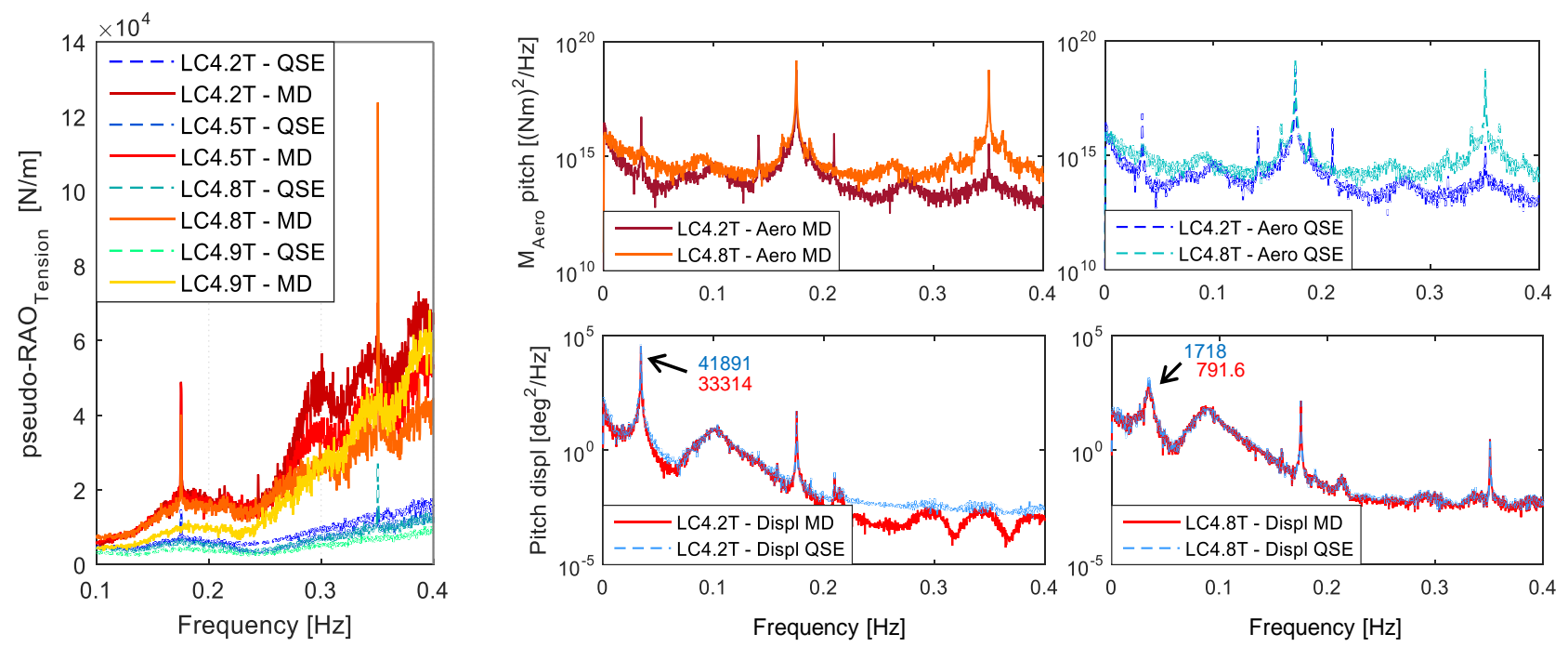

Figure 8: Mooring line tension pseudo-RAO for a range of LCs from below rated to the turbine shut-down (left), and the along-pitch PSD of aerodynamic load (top) and displacement (bottom) for below and above rated LCs, in comparison for the two-model integrated FloVAWT 


\section{Conclusions}

In the present work, a detailed study on what are the main dynamics effects to be considered, when modelling the dynamics of the mooring system of a floating vertical axis wind turbine, has been developed. A comparison between the results derived with a quasi-static (energetic) approach and MoorDyn (in use in FAST, by NREL), both coupled with FloVAWT, has been performed, focusing on the rigid body motion and on the mooring lines' tension, linked to the chain links fatigue life.

Although the quasi-static approach predicted higher averaged values of lines tension at the fairlead, MoorDyn model reported a significantly higher cumulative damage of the hour-simulations run at the different load cases considered. More specifically, up to one order of magnitude differences have been estimated, for the one-hour cumulative damage.

The reason is partially due to the higher frequency, of comparable amplitude, of the mooring lines tension and restoring forces on the platform, despite the lower mean value. These discrepancies in the results can be explained by the ability of the lumped mass tool (MD model) to account for the line mass inertia and hydrodynamic drag effects, proportional to the velocities and the accelerations of the floating system. However, while these phenomena are clearly observable in the time histories of the mooring system restoring forces, what emerged from the analysis of the front line tension PSD is the higher response of the dynamic model to the hydro- and aerodynamics excitations and loads. Therefore, the lumped-mass approach coupling showed a twofold effect: it led to slightly greater motions of the floating system compared to the rigid-lines quasi-static approach, because of the additional consideration of the lines axial stiffness, and it estimated highly oscillating and higher amplitude tension. It has then been demonstrated that a dynamic approach is necessary to carry on an accurate fatigue assessment the mooring system expected life.

In conclusion, from this preliminary analysis, it can be stated that, if the aim of the analysis is an accurate estimation of either the platform motions (in the surge and the pitch directions mainly) or the global mooring system restoring forces and moments, the additional fluctuations recorded in the MoorDyn modelled tension have to be taken into account, leading to non-negligible variations of the results. 


\section{References}

[1] Carbon Trust, 2015, Floating Offshore Wind : Market and Technology Review.

[2] Jonkman, J., and Musial, W., 2010, Offshore code comparison collaboration (OC3) for IEA task 23 offshore wind technology and deployment.

[3] Robertson, A., Jonkman, J. M., Masciola, M., Song, H., Goupee, A. J., Coulling, A. J., and Luan, C., "Definition of the Semisubmersible Floating System for Phase II of OC4."

[4] Barone, M., and Paquette, J., 2012, Vertical-Axis Wind Turbines Revisited: A Sandia Perspective.

[5] Borg, M., Collu, M., and Brennan, F. P., 2012, "Offshore floating vertical axis wind turbines: Advantages, disadvantages, and dynamics modelling state of the art," International Conference on Marine and Offshore Renewable Energy 2012, RINA, Royal Institution of Naval Architects.

[6] Paulsen, U. S., 2013, DeepWind-An Innovative Offshore Wind Turbine Concept.

[7] Parsons, D. J., Chatterton, J. C., Brennan, F. P., and Kolios, A. J., 2011, Carbon Brainprint Case Study: Novel Offshore Vertical Axis (NOVA) wind turbines.

[8] Parneix, N., Fuchs, R., Immas, A., Silvert, F., and Deglaire, P., 2016, Efficiency improvement of vertical-axis wind turbines with counter-rotating.

[9] Borg, M., and Collu, M., 2015, “A comparison between the dynamics of horizontal and vertical axis offshore floating wind turbines," Phil.Trans. Roy. Soc. A, 373(2035), p. 20140076.

[10] Wang, K., Otto, M., and Hansen, L., 2014, "Comparative study of a FVAWT and a FHAWT with a semi-submersible floater," Proceedings of the Twenty-fourth International Ocean and Polar Engineering Conference, ISOPE, Busan, Korea, pp. 302-310.

[11] Cheng, Zhengshun; Madsen, Helge AAgaard; Chai, Wei; Gao, Zhen; Moan, T., 2017, “A comparison of extreme structural responses and fatigue damage of semi-submersible type floating horizontal and vertical axis wind turbines," Renew. energy, 108, pp. 207-219.

[12] Borg, M., Collu, M., and Brennan, F. P., 2012, "Offshore floating vertical axis wind turbines: Advantages, disadvantages, and dynamics modelling state of the art," RINA, R. Inst. Nav. Archit. - Int. Conf. Mar. Offshore Renew. Energy 2012, (January).

[13] Borg, M., 2014, "Offshore floating vertical axis wind turbines: development \& applications of a coupled model of dynamics," Cranfield University.

[14] Cheng, Z., 2016, "Integrated Dynamic Analysis of Floating Vertical Axis Wind Turbines," Norwegian University of Science and Technology (NTNU).

[15] Cevasco, D., Collu, M., Hall, M., and Rizzo, C. M., 2017, “On the Comparison of the Dynamic Response of an Offshore Floating VAWT System when Adopting Two Different Mooring System Model of Dynamics: Quasi-Static VS Lumped Mass Approach," 36th International Conference on Ocean, Offshore and Arctic Engineering, ASME, Trondheim, Norway, pp. 19.

[16] Shires, A., Collu, M., and Borg, M., 2013, "FloVAWT: Progress on the development of a coupled model of dynamics for Floating offshore Vertical Axis Wind Turbines," The International Society of Offshore and Polar Engineers 2013, ISOPE.

[17] Collu, M., Borg, M., Shires, A., Rizzo, F. N., and Lupi, E., 2014, "FloVAWT: Future progress on the development of a coupled model of dynamics for a offshore VAWT," Proceedings of the ASME 2014 33rd International Conference on Ocean, Offshore and Arctic Engineering, ASME, San Francisco, California, USA, pp. 1-11.

[18] Collu, M., Manuel, L., Borg, M., and Liu, J., 2015, "Long-Term Global Response Analysis of a Vertical Axis Wind Turbine Supported on a Semi-Submersible Floating Platform: Comparison Between Operating and Non-Operating Wind Turbine Load Cases," VI International Conference on Computational Methods in Marine Engineering (MARINE), Rome, Italy.

[19] Collu, M., Borg, M., and Lance, M., 2016, "On the relative importance of loads acting on a 
floating vertical axis wind turbine system when evaluating the global system response," Proceedings of the ASME 2016 35th International Conference on Ocean, Offshore and Arctic Engineering, ASME, Busan, South Korea, pp. 1-11.

[20] Shires, A., Collu, M., and Borg, M., 2013, "FloVAWT: progress on the development of a coupled model of dynamics for floating offshore vertical axis wind turbines," Proceedings of the Twenty-third (2013) International Offshore and Polar Engineering, ISOPE, Anchorage, Alaska, USA, pp. 345-351.

[21] Jonkman, B., and Kilcher, L., 2012, TurbSim User's Guide: Version 1.06.00.

[22] Collu, M., Borg, M., Shires, A., Rizzo, F. N., and Lupi, E., 2014, "FloVAWT: Further Progresses on the Development of a Coupled Model of Dynamics for Floating Offshore VAWTs," ASME 2014 33rd International Conference on Ocean, Offshore and Arctic Engineering OMAE 2014, ASME, San Francisco, CA, June 8-13.

[23] Mertens, S., Kuik, G., and van Bussel, G., 2003, "Performance of an H-Darrieus in the skewed flow on a roof," J. Sol. Energy Eng., (125), pp. 433-440.

[24] Fossent, T. I., and Perez, T., 2010, "MSS. Marine Systems Simulator."

[25] Jonkman, J. M., 2009, "Dynamics of Offshore Floating Wind Turbines-Model Development and Verification," Wind EnergyWind Energy, 12, pp. 459-492.

[26] Wang, K., Moan, T., and Hansen, M. O. L., 2016, "Stochastic dynamic response analysis of a floating vertical-axis wind turbine with a semi-submersible floater," Wind Energy.

[27] Borg, M., Wang, K., Collu, M., and Moan, T., 2014, "A Comparison of Two Coupled Model of Dynamics for Offshore Floating Vertical Axis Wind Turbines (VAWT)," Proceedings of the ASME 2014 33rd International Conference on Ocean, Offshore and Arctic Engineering OMAE 2014, ASME, San Francisco, CA.

[28] Borg, M., Collu, M., and Kolios, A., 2014, "Offshore floating vertical axis wind turbines, dynamics modelling state of the art. Part II: Mooring line and structural dynamics," Renew. Sustain. Energy Rev., 39, pp. 1226-1234.

[29] Ma, G., Sun, L., Wang, H., and Ai, S., 2013, "Nonlinear Mechanical Model for Static Analysis of Stretched Mooring Line," 9, pp. 184-189.

[30] Goldschmidt, M., and Muskulus, M., 2015, "Coupled mooring systems for floating wind farms," Energy Procedia, 80, pp. 255-262.

[31] Borg, M., and Collu, M., 2014, "A comparison on the dynamics of a floating vertical axis wind turbine on three different floating support structures," Energy Procedia, 53(C), pp. 268-279.

[32] Hall, M., Buckham, B., and Crawford, C., 2014, "Evaluating the importance of mooring line model fidelity in floating offshore wind turbine simulations," Wind Energy, 17(April 2013), pp. 1835-1853.

[33] Karimirad, M., 2013, "Modeling aspects of a fl oating wind turbine for coupled wave e windinduced dynamic analyses," Renew. Energy, 53, pp. 299-305.

[34] Hall, M., 2015, MoorDyn User 's Guide: Version 1.0.1.

[35] Hall, M., and Goupee, A., 2015, "Validation of a lumped-mass mooring line model with DeepCwind semisubmersible model test data," 104, pp. 590-603.

[36] Vita, L., 2011, "Offshore Floating Vertical Axis Wind Turbines with Rotating Platform," PhD Thesis, Riso DTU.

[37] Wang, K., Moan, T., and Hansen, M., 2013, "A Method For Modeling Of Floating Vertical Axis Wind Turbine,” Proc. ASME 2013 32nd Int. Conf. Ocean. Offshore Arct. Eng., pp. 110 .

[38] Det Norske Veritas and Germanischer Lloyd, 2015, OFFSHORE STANDARD DNVGL-OSE301: Position mooring, rules@ dnvgl.com.

[39] Hoadley, P. W., Frank, K. H., Yura, J. A., Hoadley, P. W., and Frank, K. H., 1983, Estimation of the Fatigue Life of Structural Steel Bridge Details, Texas.

[40] Rychlik, I., 1993, "Characterization of Random Fatigue Loads," Stochastic Approach to 
Fatigue: Experiments, Modelling and Reliability Estimation, K. Sobczyk, ed., Springer Vienna, Vienna, pp. 35-78.

[41] ASTM, 2011, Standard Practices for Cycle Counting in Fatigue Analysis, West Conshohocken, Pennsylvania (USA).

[42] Jonkman, J., 2009, "Memorandum - Load Case 5.4 from OC3 Phase IV (Floating)," pp. 1-4.

[43] Borg, M., and Collu, M., 2015, "Frequency-domain characteristics of aerodynamic loads of offshore floating vertical axis wind turbines," Appl. Energy, 155, pp. 629-636.

[44] Masciola, M., Robertson, A. N., Jonkman, J. M., Coulling, A., and Goupee, A., 2013, "Assessment of the Importance of Mooring Dynamics on the Global Response of the DeepCwind Floating Semisubmersible Offshore Wind Turbine," 23th Int. Offshore Polar Eng., 9, pp. 359-368.

[45] Cheng, Z., Wang, K., Gao, Z., and Moan, T., 2015, "Dynamic response analysis of three floating wind turbine concepts with a two-bladed Darrieus rotor," J. Ocean Wind Energy, 2(4), pp. 213-222. 